Christian Eccher

Facoltà di Filosofia

Università di Novi Sad
UDC: 316.323:821.131.1-31.09 Sequi E. DOI: $10.19090 / \mathrm{gff} .2021 .1 .139-148$

Originalni naučni rad

\title{
DECOLONIZZARE LA MENTE DEGLI ITALIANI
}

In questo lavoro, ci occuperemo dell'estremo provincialismo che ha caratterizzato e caratterizza gli studi di italianistica. L'Italia, infatti, è un paese che ha perso il passo con la modernità già nel XV secolo, quando il resto d'Europa si apriva al fenomeno della „mondializzazione“. Dimostreremo come, da allora, l'Italia si sia rifugiata nel mito della Classicità e del Rinascimento, da cui non è riuscita a uscire neanche dopo che il paese si è unito in un'unica entità statuale. La grande emigrazione della fine del XIX e dell'inizio del XX secolo ha contribuito a sprovincializzare molti italiani, ma non l'arte, la letteratura e la critica letteraria. Questa, poi, ha fatto di tutto per rimuovere il periodo e le atrocità coloniali italiane in Africa. Nel secondo Dopoguerra, mentre in tutta Europa cominciava a prendere piede la critica al colonialismo, la borghesia italiana (che deteneva il potere economico, politico e culturale) preferiva ignorare il proprio scadaloso operato nel corno d'Africa, in Grecia e in Albania. Allo stesso tempo, ignorava la viva letteratura di frontiera, quella istriana. Proprio per questo motivo, nella seconda parte di questo lavoro ci occuperemo del romanzo dell'istriano di adozione Eros Sequi, "Le case di Pothia" uno spaccato sulla realtà del colonialismo italiano nel Dodecaneso e sulla società istriana e jugoslava degli anni Cinquanta del secolo scorso.

Parole chiave: Decolonizzazione, mondializzazione, letteratura, borghesia, Sequi.

\section{BREVI RIFLESSIONI SULLO STATO DELLA CRITICA LETTERARIA ITALIANA}

La critica letteraria italiana, così come quella storiografica, soffre di un complesso che può essere paragonato a quello della rimozione psicanalitica. $\mathrm{Ci}$ sono argomenti, infatti, di cui non solo la critica letteraria italiana, ma anche la letteratura non si sono mai occupate, nonostante siano di fondamentale importanza per definire l'identità nazionale. Il principale argomento oggetto di rimozione freudiana è senza dubbio il periodo coloniale italiano, quello in cui la "laida e meschina italietta", per rubare un verso a Giorgio Caproni, decise di diventare potenza mondiale e di reclamare il proprio posto nello scacchiere mondiale del

christian.eccher@tiscali.it 
colonialismo. Ci sono solo due romanzi che si occupano di quel periodo: "Tempo di uccidere" di Ennio Flaiano e "Le case di Pothia" di Eros Sequi, di cui ci occuperemo nella seconda parte di questo lavoro. Anche a livello storico, il solo studio che si occupi a tutto tondo del colonialismo italiano, e non solo a livello militare, è "Oltremare" di Nicola Labanca (2007). È stato proprio Labanca il primo a sottolineare che la prima grande emigrazione italiana non fu quella verso l'America, ma verso l'Africa, e questo nell'immediato periodo post-unitario, quando l'Italia non aveva ancora le proprie colonie (Labanca, 2002: 57-128). Questa storia mai narrata rivela il volto niente affatto laido e meschino degli italiani: nelle colonie francesi e inglesi, soprattutto nei paesi del Maghreb, andavano commercianti, avventurieri, antropologi, missionari, lavoratori, che senza pregiudizi e con genuina curiosità, si aprivano alla diversità, cercavano di comprendere i mondi in cui la povertà, la necessità o la sete di sapere li avevano spinti. Di questa interessante e bella pagina della Storia nazionale italiana, gli intellettuali del Belpaese non si sono mai voluti occupare. Hanno voltato categoricamente la testa, nei libri di storia scolastici non c'è traccia di questa emigrazione; i libri scolastici riflettono lo stato della ricerca a livello universitario. Allo stesso modo, i critici letterari si sono nettamente rifiutati di far pubblicare e analizzare le opere degli autori italiani d'Istria, che hanno dagli anni Settanta i poi hanno dato vita a una produzione originale, a cavallo fra due culture, quella italiana e quella degli slavi del sud ${ }^{2}$. L'Istria è per l'Italia quello che per la Serbia rappresenta il Kosovo: una terra persa dopo il Trattato di Parigi del 1948 il cui ricordo le istituzioni romane hanno preferito, ancora una volta, rimuovere completamente. Solo negli anni Novanta, grazie alla scuola del comparatista Armando Gnisci (attenzione, grazie a

\footnotetext{
${ }^{1}$ Ennio Flaiano, Tempo di uccidere, Longanesi, Milano, 1947.

${ }^{2}$ Alla casa editrice Einaudi, legata a doppo filo al PCI (Partito Comunista Italiano), Natalia Ginsborg censurava senza pietà i libri degli italiani d'Istria. Aveva ricevuto ordine tassativo dal PCI, in questo in totale accordo con la DC (Democrazia Cristiana), di non far pubblicare gli autori istriani. Il PCI non voleva che il proprio elettorato sapesse come si viveva realmente in un paese comunista, la DC non perdonava ai 30.000 italiani d'Istria di essere rimasti in Jugoslavia e di non avere scelto la strada dell'esodo come i restanto 300.000 loro connazionali. L'autore di questo articolo è in possesso di testimonianze scritte che evidenziano la censura operata nei confronti degli autori istriani, testimonianze che verranno pubblicate in lavori futuri. I documenti relativi all'Istria, agli istriani e agli autori istriani sono stati bruciati nella sede di Botteghe Oscure, come ben testimoniato da diverse persone nel libro di Andrea Berrini "Noi siamo la classe operaia" (Berrini, 2004: 42).
} 
un comparatista, non a un italianista), la critica si è aperta e ha cominciato a occuparsi anche delle tematiche che erano tabù, almeno per quel che riguarda il colonialismo: l'Istria deve ancora aspettare, di questo scabroso argomento si occupano solo nelle Università di Udine e di Trieste e di Vienna (Eccher, 2012: 31-37) ${ }^{3}$. Dobbiamo però chiederci perché tutto ciò è avvenuto: alla base della rimozione c'è sempre un trauma, qualcosa che ha turbato lo sviluppo dell'individuo - o di un gruppo sociale - nel corso dell'infanzia. Che trauma ha minato la base dell'immaginario collettivo italiano? Il fascismo prima e la sconfitta subita da parte degli Alleati durante la Seconda Guerra mondiale poi sono sicuramente reponsabili della rimozione, ma solo in parte. Il vero terremoto psicologico per gli intellettuali italiani è avvenuto nel periodo più florido della storia culturale della penisola, durante il Rinascimento, quando i principati e i loro abitanti erano al culmine del proprio sviluppo artistico, tecnologico ed economico. Mentre l'Italia, come entità culturale e geografica e non certo nazionale, dominava l'Europa grazie ai suoi artisti e ai suoi scienziati, i portoghesie gli spagnoli (seguiti ben presto da olandesi, inglesi, francesi) navigavano gli oceani ed entravano in contatto con nuovi e popoli e nuove culture. Un contatto che ben presto diventerà scontro, ma che ha cambiato per sempre la coscienza dell'Europa. Cominciava quella che viene definita come "mondializzazione" (Gnisci, 2009: 9-18). Nel secolo scorso, in epoca di decolonizzazione e soprattutto in Francia e in Inghilterra, lo scontro si è tradotto, almeno a livello culturale, in una profiquacritica all'eurocentrismo e in un'apertura alle culture e alle letterature dei mondi. L'Italia, fino agli anni Novanta del Novecento, è rimasta fuori da questo processo, estranea alla globalizzazione, prigioniera dell'immaginario - per usare la terminologia dello pischiatra e filosofo Jacques Lacan (Lacan, 2016: 77) - legato al Rinascimento e quindi alla Classicità, dato che le premesse teoriche dell'Umanesimo affondano proprio nel recupero dei valori artistici del periodo ellenico e romano. L'Italia ha visto sé stessa attraverso gli occhi degli stranieri, attraverso le descrizioni e i ritratti che del paese - anzi, dei paesi che all'apoca la componevano - tracciavano i rampolli dell'aristocrazia e della borghesia nordeuropea durante i loro Grand Tour lungo lo stivale (Gnisci, 2009: 17). Gli intellettuali italiani non sono ancora riusciti a uscire dall'immagine dell'Italia intesa come crogiuolo di classicità, nonostante i numerosi studi genetici (Cavalli-Sforza, 2001: 10-56), iconologici e culturali (Matvejević, 1987) che tendono a collocare il paese non in un lontano passato storico, ma in una geografia moderna di incroci e di incontri: l'Italia

\footnotetext{
${ }^{3}$ Ci sono convegn i tenuti a Trieste e organizzati dall'Università Cattolica di Milano su questo argomento, convegni che però hanno uno stampo spiccatamente nazionalista. L'analisi degli atti dei convegni tenuti da IRCI saranno oggetto di un futuro lavoro.
} 
non è (solo) il paese della Classicità, ma è anche, e soprattutto, un mondo in mezzo ai mondi, bagnato dal Mediterraneo e quindi in contatto con tutte le culture connesse al Mare Nostrum (Gnisci, 2009: 9). A nord della penisola ci sono città come Trieste o Gorizia che appartengono non solo al Mediterraneo e all'Italia, ma anche al mondo tedesco. Come è possibile considerare Carlo Michelstaedter un filosofo e uno scrittore puramente italiano? I romanzi di Italo Svevo hanno sicuramente più connessioni con la letteratura e la cultura austriaca (da Freud a Zweig) che non con quella italiana.

Quale prospettiva dovrebbe allora seguire la critica letteraria italiana per uscire dal proprio pantano nazionale e per aprirsi ai mondi che la compongono, in maniera tale da superare (rivalutandolo in una nuova luce!) il retaggio del Rinascimento e della Classicità, che indubbiamente esiste? Come fare per decolonizzare la mente degli italiani, che si ritengono gli eredi diretti dei geni rinascimentali e nutrono un senso di superiorità nei confronti delle altre culture e degli altri popoli? L'unica soluzione è a mio avviso quella di aprirsi alla comparatistica, e in particolare al metodo indicato da studiosi come NgugiwaThiong'o (Thiong'o: 2000), che propone di spostare il centro del mondo togliendo così il primato all'Europa, e soprattutto tracciato da DionýzĐurišin e da Armando Gnisci nella loro opera fondamentale "Il Mediterraneo: una reteinterletteraria". Il Centrismo interletterario è costituito da un insieme di letterature nazionali che presentano fra loro numerose affinità. L'Italianistica ha un futuro soltanto se diventerà finalmente strabica: con un occhio deve guardare alla produzione letteraria in lingua italiana, con l'altro al resto del mondo, e trovare i fili segreti che ci porteranno a tessere la tela della letteratura mondiale. Da questo punto di vista, ci potrà essere utile l'analisi della letteratura degli italiani d'Istria, che di per sé si colloca a cavallo fra tre centrismi interletterari: quello dell'Europa occidentale, quello mediterraneo e quello degli slavi del sud. A questo proposito, analizzeremo l'opera di uno dei due autori italiani che, non a caso, si sia occupato del colonialismo italiano: Eros Sequi, toscano trasferitosi in Istria durante la Seconda guerra mondiale con il sogno di aiutare gli jugoslavi a edificare lo Stato socialista e rimasto per sempre a vivere nella ex Jugoslavia.

\section{LE CASE DI POTHIA}

ErosSequi pubblicòLe case di Pothianel 1957. Nel suo romanzo, gli italiani sono emblema di sfruttamento e di colonialismo. Pothia è l'unico villaggio dell'isola di Kalymnos, nel Dodecaneso, un'area sulla quale il governo italiano aveva esteso un protettorato che comprendeva quattordici isole delle Sporadi meridionali, dopo averle conquistate fra il 26 aprile e il 12 maggio del 1916. Si è discusso a lungo se la presenza degli italiani nelle Sporadi avesse connotazioni coloniali o meno. L'Italia aveva perso 
infatti molto presto l'interesse nei confronti di queste isole di cui aveva reclamato il possesso al fine di spingere Istanbul a firmare una pace che sancisse il predominio di Roma sulla Libia; la fine della prima guerra mondiale aveva portato via con sé l'impero turco, di cui rimaneva solo un piccolo stato islamico, la Turchia appunto. La presenza italiana nelle Sporadi fu così marginale, ridotta a un mero avamposto militare nel mar Mediterraneo. Come ben ricorda Nicola Labanca in Oltremare(Labanca, 2002: 178183), però, i governatori di epoca fascista Mario Lago e Cesare Maria De Vecchi attuarono politiche di assimilazione e di repressione nei confronti della popolazione locale, tipiche di un protettorato coloniale. Il vero problema non sta, a mio avviso, nello stabilire se gli italiani abbiano adottato politiche coloniali in Eritrea e Somalia piuttosto che nell'Egeo. Il nocciolo della questione, e il secondo libro di Sequi ci dà lo spunto per comprendere ciò, sta nel fatto che le istituzioni e l'intellettualità italiana non hanno fatto altro che rimuovere, in senso freudiano, il proprio passato coloniale, come abbiamo già osservato precedentemente. Come se l'Italia non si fosse macchiata di ignobili efferatezze in Eritrea, in Somalia e in Albania. La rimozione passa attraverso un pregiudizio molto diffuso, racchiuso nell'espressione "italiani brava gente" che tende a considerare quello italiano un colonialismo all'acqua di rose. Mi limito qui a ricordare i massacri in Libia e le deportazioni di popolazioni inermi, i bombardamenti aerei ordinati dal generale Graziani sui villaggi ribelli dell'Africa italiana ${ }^{4}$.

Almeno nei primi capitoli, Le case di Pothiaè una rievocazione di un mondo povero, e la dominazione italiana si inserisce in un contesto di estrema miseria e rassegnazione.

Sequiera nato nel 1912 e a 23 anni fu mandato proprio nelle Sporadi come insegnante di lingua italiana nel locale liceo. Le case di Pothia è un testo tessuto sulla delicatezza di lontani ricordi, costituito da diversi capitoli collegati fra loro non da una trama intesa in senso classico: ogni capitolo racconta una vicenda particolare e a essere protagonista è la miseria che si annida fra il mare e le case gialle affacciate su una piccola baia. La Storia rimane sullo sfondo: i personaggi si agitano su uno scenario apparentemente immutabile, in cui l'oppressione e lo sfruttamento sembrano essere le

\footnotetext{
${ }^{4}$ La Libia ha istituito una "giornata della memoria" per ricordare la deportazione nel centro del deserto delle popolazioni che abitavano sulla costa. Il colonialismo italiano in Libia è il soggetto di un film del 1980, mai proiettato in Italia perché censurato, "Il leone del deserto", realizzato nel 1980 dal regista siriano MustafaAkkad con Oliver Reed nel ruolo di Rodolfo Graziani. I bombardamenti del 1936 sull'Eritrea e sulla Somalia furono i primi a essere effettuati grazie a degli aeroplani, e aprirono una nuova e triste era nelle tecniche di annientamento bellico.
} 
condizioni normali in cui gli abitanti dell'isola trascorrono la propria vita. Il racconto si concentra sulle condizioni economiche e sui costumi di questi, «i quali non sono pescatori come altri. Da tempo immemorabile portano alla banchina di Pothia le spugne viscose, che altri lavorano per mandarle ai grandi mercati dell'Inghilterra. (...) È di Kalymnos il re delle spugne: che abita a Londra e ha tre ville nell'isola natale; e tanti pescatori pescano per lui anche nel mare dei Caraibi. In primavera, sul recinto della villa di Pothia, fiorisce la passiflora. Ma i poveri cristi sono i calinioti». (Sequi, 1979: 42) Non c'è apparentemente pathos nella descrizione, costituita da frasi paratattiche che sembrano non lasciare spazio alla commozione. Con estrema lucidità narrativa lo scrittore racconta dei pescatori che, dopo aver ricevuto l'acconto, si nascondono dentro casa per non partire alla volta della pesca alle spugne. Le autorità dell'isola, i carabinieri italiani in primo luogo, stanano i pescatori che, costretti a imbarcarsi, staranno in mare aperto dalla primavera fino all'autunno; il loro lavoro è pericoloso. Si tratta infatti di entrare in uno scafandro, raggiungere il fondo marino e di rimanervi lunghi minuti a raccogliere spugne. La risalita, se effettuata troppo in fretta, può causare una paralisi fortemente invalidante; è così che in autunno, a raccolta finita, le navi vomitano decine e decine di paralitici. Chi non è incorso nella disgrazia, ha il tempo di lasciare incinta la moglie che, consumati i soldi dell'acconto, è stata magari costretta a sfamare i figli avuti precedentemente concedendosi carnalmente al proprietario dell'unica merceria del villaggio. Malato per giunta di sifilide. I tratti realistici si perdono all'inizio del terzo capitolo, intitolato Il mare vermiglio, in cui l'autore spiega anche le ragioni che l'hanno spinto a scrivere di Kalymnos:

«Sfiniscono tutte queste voci assordanti di gente fra le sbarre; riapro gli occhi.

Il Quarnero abbracciato dal monte Maggiore e il profilo azzurrino di Cherso. Oppure, il mare di Kalymnos, stretto dai monti rosati di Pothia. In fondo naviga nell'Egeo Coocinestrina.

A occhi aperti, dal coro salta fuori una figura chiara a ripetermi la storia, che già ho dentro da tanti anni: devo raccontarla, mentre tintinna negli orecchi la campanella di San Nicola dei Palombari. Quando avrò fatto sapere a tutti la storia di Cleantis e di Nichi, me ne sarò liberato il respiro». (Sequi, 1979: 41)

Il bozzetto realistico cede improvvisamente il posto a un intermezzo che fa riferimento alla situazione politica degli anni Cinquanta. Perché l'autore ha sentito solo allora, dal Quarnero, nel momento in cui il sogno socialista per lui si stava spegnendo, l'esigenza di raccontare la storia dei pescatori di Kalymnos? A cosa era dovuta la sensazione di soffocamento che il non aver mai riferito tali avvenimenti comportava? Forse all'oppressione sociale e politica che si respirava in Istria e in 
Jugoslavia, dove viveva lo scrittore? Probabilmente sì. Nel 1948, Tito aveva rotto con Stalin: la Jugoslavia si era rifiutata di diventare un paese satellite dell'Unione Sovietica e di produrre i beni che servivano ai russi. Lo scisma jugoslavo causò un'ondata di paura nel paese degli slavi del sud, paura non del tutto ingiustificata: le autorità di Belgrado avevano paura di un attacco militare da parte dell'URSS e dei suoi alleati, in partcolare di quelli confinanti, la Romania e l'Ungheria. Le minoranze nazionali, a cominciare da quella italiana che ancora portava la stigmata del fascismo, erano considerate quinte colonne al servizio delle potenze straniere. Negli anni Cinquanta, la componente italiana dell'Istria fu ridotta al silenzio con ogni mezzo (Eccher 2012, 19-26). In Eravamo in tanti, Sequi collegava la propria lotta con la liberazione dei kalimnioti da ogni tipo di oppressione:

«E questa nostra lotta, e queste miserie? Un giorno, le mamme di Calino daranno il pane ai figliuoli, e non più al papasso barbuto». (Sequi, 1959: 90)

Le mamme dei bambini, che offrivano i propri risparmi a San Nicola con la speranza che questi riportasse a casa vivo il marito lontano, avrebbero non solo continuato a offrir danaro al papasso, ma anche a fare la vita misera di sempre, e i bambini malati di tracoma a scorazzare fra le case gialle di Pothia in un ambiente malsano, e i mariti a tornare paralizzati dai fondali del mare Egeo. Nessun nuovo mondo sarebbe stato realmente costruito, per i pescatori di Kalymnos non ci sarebbe stata nessuna salvezza. La società sognata da Sequi e dai partigiani era destinata a non nascere. Nelle Case di Pothia, Il sogno di Sequi è diventato un incubo, da cui l'autore aspira a liberarsi tramite la letteratura. Quest'ultima non coincide più con la vita, ma rimane, nel racconto della realtà degli umili, una forma di riscatto, che non può però incidere in alcun modo sulla società. Sequi si limita a ricordare, e il ricordo del pescatore Nichi e della moglie Cleantis assume sulla pagina immagini oniriche e vagamente espressioniste:

«Così vedo Nichi, sul molo, con la mano al petto, il bimbo; entra nel buio, si dissolve. Mi ritorna incontro la massa di figure urlanti, mi si accalca, mi leva il fiato; tante Nichi, tanti Cleantis, onde di fiori di mandorlo, aranceti, acqua buia, letame di fogne, arsura, fame, scintillìo di riflessi. Télendo sospesa rosata sulle acque, sporcizia. E Nichi è sparita dalla folla buia, torna a casa col suo uomo, poi l'uomo riparte, il bimbo s'ammala, la mamma ha il petto vuoto, i soldi finiscono.

Riemerge Nichi, la moltitudine sparisce, la passiflora china la corona sull'inferriata della villa (...).

Perché scappa via? Già, me lo dicono, che ha salvato il piccolo a prezzo del suo amore. La folla va e viene a ondate, poi si ritira e lascia a terra Cleantis, che 
cammina tutto storto e cerca Nichi per dividere la miseria. E Nichi non ha il coraggio per togliergli anche l'illusione dell'amore.

Affogo, affogo, mi soffoca la marea di uomini, grandi e piccoli, con gli occhi smisuratamente grandi, tutti gridano, non piangono; ma è un orrore, l'orrore ha assorbiti Nichi e Cleantis e il loro figlio.

Il coro spaventoso cresce, sparisce ogni voce d'uomo, è un mare senza onde, tutto sconquassato. Io navigo su una barca, fra Télendo e Scalias, il sole è sorto dall'altra parte dell'isola, e qua l'acqua si tinge di rosso, diventa densa, vermiglia; è un orrore, è sangue. Ho ribrezzo, spavento. Perché questo ricordo? Non c'erano altri uomini sul mare di sangue, e l'orrore durò qualche istante. E giro gli occhi, mi voglio liberare. Mi libero. Nichi, Clenatis, tutta la folla, galleggiano nel sangue, ne chiudono un fiotto con i loro contorni trasparenti, vi si sciolgono come una scaglia di neve».(Sequi, 1979: 55-56)

Il mare vermigliosegna il passaggio da una Weltanschauung della letteratura fondata sulla possibilità di congiungere letteratura e vita, a un nichilismo artistico, che si traduce nell'anarchia dei generi letterari e nell'assenza di un principio-guida narrativo e di un progetto unificante. Gli ultimi quattro capitoli del romanzo ritornano al frammento, alla prosa d'arte, alla narrazione onirica ${ }^{5}$. Il frammento era la forma prediletta dagli autori che facevano riferimento alla rivista la Voce, perché meglio di tutte si adattava al clima problematico degli inizi del Novecento e alla crisi culturale che introduceva perturbamenti e incertezze. Dal punto di vista sociale l'intellettuale non sapeva dove collocarsi: aveva perduto la propria organicità e il proprio ruolo. Lo scrittore poteva allora raccontare il mondo soltanto in maniera frammentata, cambiando il punto di vista della narrazione che si presentava episodica e relativa, senza possibilità di assumere la coerenza e la compattezza del romanzo ottocentesco. Anche Sequi nelle Case di Pothiapassa dalla narrazione in terza persona, oggettiva, a quella del ricordo in prima persona. Negli ultimi capitoli, poi, la trama del racconto si perde completamente. I personaggi negativi non hanno un volto preciso, gli umili sembrano affossati nelle loro sofferenze e lì rimanere. Nessuna guerra li ha liberati, nessun partigiano li libererà. Le spugne vengono vendute sul mercato di Londra, che negli anni Trenta esercitava sull'area dell'Egeo un notevole controllo a livello economico. La

\footnotetext{
${ }^{5} \mathrm{Il}$ romanzo è composto in tutto da nove capitoli. Al mare vermiglio seguono La Grazia e $E$ domani? che riprendono la narrazione realista e lineare delle vicende degli abitanti di Pothia cominciata nei primi due capitoli e in parte interrotta dagli intermezzi in prima persona del Mare vermiglio.
} 
passiflora cresce rigogliosa sulle inferriate della villa del mercante di spugne e i poveri cristi restano tali. Nella Jugoslavia del dopoguerra era accaduto qualcosa di simile. Sequi, dall'esilio belgradese, pur dedicandosi solo sporadicamente alla scrittura, ha diretto la rivista“la battana"; malvisto dalla Comunità italiana perché troppo vicino ai quadri del regime, era contemporanemente sospettato dai dirigenti del PCJ in quanto in continuo contatto con gli italiani d'Istria. È questo il triste destino degli italiani d'Istria, per anni ignorati della autorità jugoslave, che li considerava fascisti, e da sempre dimenticati dall'Italia, che li ha considerati per anni il simbolo di una sconfitta storica da rimuovere.

\section{CONCLUSIONI}

L'analisi dell'opera di Sequi dimostra che la letteratura italiana istriana costringe il critico ad ampliare il proprio sguardo, a considerare non solo le vicende storiche e letterarie del proprio paese ma anche quelle dei paesi vicini. L'Istria e la sua cultura, ancora sconosciute in Italia, soprattutto per quel che riguarda la letteratura in lingua croata e in dialetto čakavo ${ }^{6}$, possono essere un buon punto di partenza per decolonizzare la mente degli italiani e per uscire da un'ideologia - non solo letteraria, ma anche politica - asfitticamente nazionale e nazionalista.

\section{Cristian Eccher}

\section{DECOLONIZE THE MIND OF ITALIANS}

\section{Summary}

This work deals with the extreme provincialism that has characterized and characterizes Italian studies. Italy, in fact, is a country that has lost pace with modernity already in the fifteenth century, when the rest of Europe was opening up to the phenomenon of "globalization". We have demonstrated how, since then, Italy has taken refuge in the myth of Classicism and the Renaissance, from which it has not managed to get out even after the country has joined in a single state entity. The great emigration of the late nineteenth and early twentieth century helped to de-provincialize many Italians, but not art, literature and literary criticism. Criticism did everything to remove the period and the Italian colonial atrocities in Africa. In the second post-war period, while the criticism of colonialism was

${ }^{6}$ Un valido e proficuo tentativo di incontro fra le diverse anime, culturali e linguistiche, della letteratura istriana, è quello messo in atto da Mirko Sebić nel suo progetto editoriale "Naša mesta“ (Naša mesta: geografija, sećanja i zaborava: 2014). 
beginning to take hold throughout Europe, the Italian bourgeoisie (which held economic, political and cultural power) preferred to ignore its deadline operated in the Horn of Africa, Greece and Albania. At the same time, he ignored the lively frontier literature, the Istrian one. Precisely for this reason, in the second part of this work we have dealt with the novel by the Istrian of adoption Eros Sequi, "Le case di pothia.

Keywords: Decolonization, globalization, literature, bourgeoisie, Gnisci.

\section{BIBLIOGRAFIA}

Berrini, A. (2004). Noi siamo la classe operaia. Baldini e Castoldi Dalai: Milano.

Cavalli-Sforza, L., a cura di (2001). Le radici prime dell'Europa. Einaudi: Torino.

Đurišin, D., Gnisci, A., a cura di (2000). Il mediterraneo, una rete interletteraria.Bulzoni: Roma.

Eccher, C. (2012). Laletteratura degli italiani d'Istria e di Fiume dal 1945 a oggi. Fiume-Rijeka: Edit.

Flaiano, E. (1947). Tempo di uccidere. Longanesi: Milano.

Gnisci, A. (2009). L'educazione del te. Roma: Sinnos.

Lacan, J. (2016). Seminario VII. Einaudi: Torino.

Labanca, N. (2002). Oltremare. Bologna: Il Mulino.

Matvejević, P. (2006). Breviario Mediterraneo. Milano: Garzanti.

Milani, N., Dobran, R. (2010), Le parole rimaste. Fiume-Rijeka: Edit.

Sequi, E. (1950). Eravamo in tanti. Fiume- Rijeka: Edit.

Sequi, E. (1979). Le case diPothia.Fiume-Rijeka: Edit. 